Wien klin Mag 2016 · 19:48-54

DOI 10.1007/s00740-016-0097-2

Online publiziert: 28. Januar 2016

(c) The Author(s) 2016. This article is available

at SpringerLink with Open Access

CrossMark

Das komplett künstliche Herz [,total artificial heart" (TAH)] CardioWest ${ }^{\mathrm{TM}}$ folgt dem radikalsten Konzept eines mechanischen Herzersatzes. Nach fast vollständiger Entfernung des eigenen, schwerst erkrankten und somit insuffizienten Herzens werden orthotop zwei Pumpkammern in den Thorax implantiert und über Druckleitungen mit dem externen pneumatischen Antrieb verbunden. Herzminutenvolumina über 9 I ermöglichen eine volle kardiozirkulatorische Unterstützung. Als einziges TAH, das CE-zertifiziert und von der US Food and Drug Administration zugelassen ist, findet dieses System mittlerweile v. a. in Europa und den USA weite Verbreitung. Es wurde mehr als 1400 Mal erfolgreich implantiert und ist somit trotz seines kompromisslosen Ansatzes Teil der klinischen Routine geworden.

Patienten mit weit fortgeschrittener Herzinsuffizienz im Stadium IV nach Klassifikation der New York Heart Association (NYHA) haben eine extrem schlechte Überlebensprognose und nur noch wenige erfolgreiche Behandlungsoptionen [11]. Medikamentös kann ihnen derzeit kein längerfristiges Behandlungskonzept angeboten werden, das Lebensqualität und Langzeitüberleben signifikant verbessern würde [3]. Signifikant bessere Überlebenschancen bieten derzeit nur chirurgische Optionen, die orthotope Herztransplantation (HTX) als biologischer Herzersatz $[1,6,18]$ und die mechanische Kreislaufunterstützung

Dieser Beitrag wurde in der Zeitschrift Med Klin Intensivmed Notfmed 2015 • 110:431-437. doi:10.1007/s00063-015-0060-9 erstveröffentlicht. Zweitpublikation mit freundlicher Genehmigung des Autors.

Herwig Antretter · Julia Dumfarth - Daniel Höfer

Universitätsklinik für Herzchirurgie, Department Operative Medizin, Medizinische Universität Innsbruck, Innsbruck, Österreich

\title{
Das komplett künstliche Herz
}

bzw. der Herzersatz, die sog. „ventricular assist devices" (VAD) und TAH $[6,12]$.

\section{Herztransplantation}

Die HTX ermöglicht beste Ergebnisse für Patienten im Stadium NYHA IV bezüglich des Kurz- und Langzeitüberlebens [1, 18] und auch hinsichtlich der Lebensqualität [6]. Allerdings sind alle Transplantationszentren weltweit mit identischen Problemen konfrontiert: mit einem eklatanten Mangel an guten Spenderorganen in Relation zur Zahl potenzieller Empfänger sowie mit einer mehrere Monate dauernden Wartezeit auf ein lebenserhaltendes Spenderorgan, die einige potenzielle Organempfänger nicht überleben.

\section{Mechanische Unterstützungs- systeme}

Daher wurden mechanische Herzunterstützungssysteme entwickelt - initial in der Indikation „bridge to transplant", um Patienten zu stabilisieren, deren Zustand sich auf der Warteliste zur Transplantation sukzessive verschlechterte. Nach Implantation eines VAD konnte der Patient von den meist präoperativ benötigten Katecholaminen entwöhnt, mobilisiert und bis zum Eintreffen eines akzeptablen Spenderherzens etliche Monate bei guter, oft sehr hoher Lebensqualität auch außerhalb der Klinik stabil und mobil gehalten werden [20].

Einige Patienten konnten nach Ausheilung der Entzündung und Erholung des Herzmuskels bei wieder normaler Pumpfunktion von der mechanischen Kreislaufunterstützung entwöhnt werden („bridge to recovery“; $[9,15])$. Meist war dies bei einem kurzen Krankheitsverlauf der Fall, v. a. bei Myokarditiden.
Die Entwicklung modernster, elektrisch betriebener Rotationspumpen, genauer Axial- und Zentrifugalpumpen, als linksventrikuläre VAD ermöglicht heute so gute Kurz- und Langzeitergebnisse, dass sich eine neue Indikation ergab: die „alternative to transplant“ (oder „chronic implantation“ bzw. „destination therapy“; [16]). Patienten an diesen Systemen werden nicht mehr bezüglich einer HTX evaluiert, eine mehrjährige Unterstützung bei hoher Lebensqualität ist möglich, die längste Augmentationszeit beträgt bisher über 8 Jahre. Allerdings sind diese Systeme nur für den linksventrikulären Einsatz zugelassen. Die überwiegende Mehrheit der derzeit weltweit implantierten Assistenzsysteme unterstützt mechanisch ausschließlich den versagenden linken Ventrikel [2].

\section{Das komplett künstliche Herz}

Das TAH CardioWest ${ }^{\mathrm{Tm}}$ der Firma SynCardia Systems, Inc. (Tuscon, AZ, USA) verfolgt einen gänzlich anderen Ansatz, kommt bei selektiveren Indikationen zum Einsatz und wird bis auf Weiteres in Relation zur Gesamtzahl aller jährlich implantierten mechanischen Kreislaufsysteme im niedrigen Prozentbereich reüssieren. Trotzdem fasziniert das Konzept eines echten Kunstherzens anstelle des eigenen Herzens gleichermaßen die Öffentlichkeit wie die medizinische Fachwelt [5].

Das TAH CardioWest ${ }^{\mathrm{Tm}}$ wurde von den Doktoren Kolff, Olsen und Jarvik zwischen 1950 und 1970 designt, entwickelt und präklinisch im Tierversuch getestet, all das mit dem Ziel eines permanenten mechanischen Herzersatzes. 


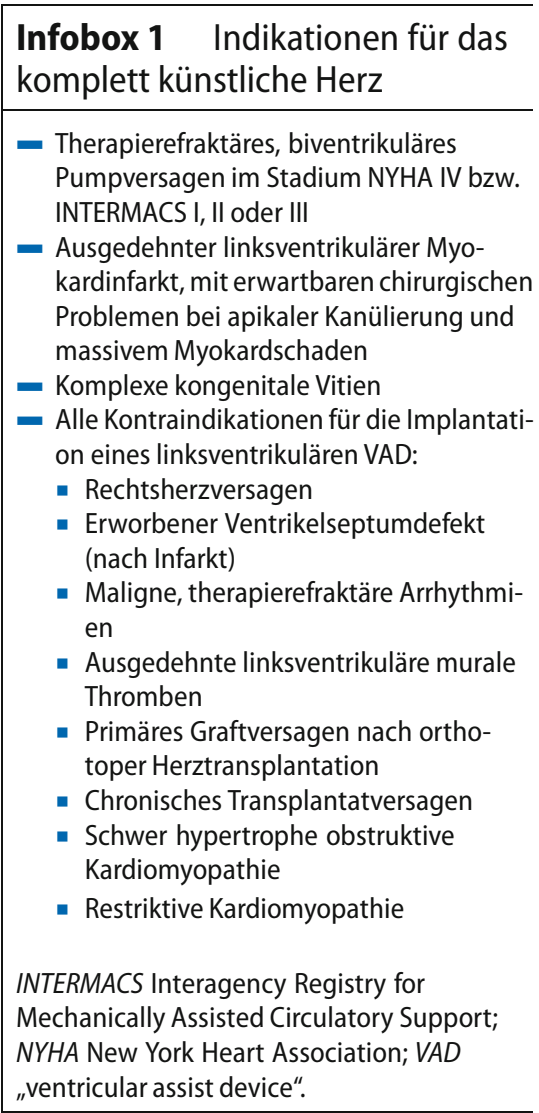

\section{Aufbau}

Das TAH ist eine orthotope, pneumatisch angetriebene, pulsatile polyurethanbeschichtete Pumpe bestehend aus zwei Ventrikeln, die den menschlichen rechten und linken Ventrikel ersetzen. Drahtverstärkte Druckleitungen, die durch die Bauchdecke ausgeleitet werden, verbinden die Pumpkammern mit der Antriebskonsole. Mit dem etwa 6 kg schweren „Freedom Driver“ ist ein portabler pneumatischer Antrieb verfügbar, der dem mobilisierten Patienten maximale Freiheit und damit die Entlassung aus der Klinik ermöglicht. Das Implantat im Thorax wiegt $160 \mathrm{~g}$ und hat ein Gesamtvolumen von ungefähr $400 \mathrm{ml}$.

Beide Ventrikel sind gleich aufgebaut, obwohl sie sich in der Anordnung und Angulation der Inflow- und OutflowKlappen aufgrund der unterschiedlichen anatomischen Verhältnisse für den rechten und linken Ventrikel unterscheiden müssen. Die Klappen waren bisher mechanische Pyrolyt-Carbon-Einschei- benklappen von Medtronic-Hall, sind aber von SynCardia aufgekauft worden und werden nun unter dem Namen SynHall $^{\text {sw }}$ produziert (Inflow-Prothese $27 \mathrm{~mm}$; Outflow-Prothese $25 \mathrm{~mm}$ für den $70 \mathrm{~cm}^{3}$-Ventrikel).

\section{》) Mit dem TAH wird ein Herzminutenvolumen von über 9 l erreicht}

Im Ventrikel trennt eine 4-lagige impermeable Membran die „Blutkammer“von der „Luftkammer“. Während der Ejektionsphase wird komprimierte Luft in die Luftkammer gepresst, dadurch drückt die Membran gegen die gefüllte Blutkammer und wäscht das Blut durch die Ausflussklappe in die Zirkulation. In der Füllungsphase zieht ein Vakuum die Membran in die Gegenrichtung und ermöglicht damit die Füllung der Blutkammer über die nun geöffnete Einlassprothese. Die maximale Exkursion der Membran erlaubt damit den Auswurf von bis zu $70 \mathrm{ml}$ Blut pro Zyklus, so wird ein Herzminutenvolumen von über 91 erreicht.

\section{Indikationen}

Die überwiegende Zahl herzinsuffizienter Patienten mit mechanischer Überbrückung kommt mit einem linksventrikulären VAD (LVAD) aus. Einige benötigen auch eine rechtsventrikuläre Unterstützung (RVAD), diese biventrikulär unterstützten Patienten haben aber ein höheres perioperatives Risiko [8] und einen schlechteren Langzeitverlauf: Das 1-Jahres-Überleben beträgt $55 \%$ gegenüber $80 \%$ bei Patienten mit alleinigem LVAD [13].

Bei Vorliegen eines schweren biventrikulären Versagens ergeben sich verschiedene Optionen: einerseits die orthotope HTX als Goldstandard, andererseits die mechanische Unterstützung mithilfe biventrikulärer pulsatiler parakorporaler oder implantierbarer Systeme oder des TAH [17]. Vorteile des TAH liegen im hohen Pumpvolumen, dem Fehlen von Limitationen aufgrund von Inflow- oder Outflow-Kanülen, der sofortigen Reduktion an Inotropika bzw. Vasokonstriktiva und damit der Vermeidung deletärer va- sokonstriktorischer Effekte sowie einer raschen Mobilisation des Patienten.

\section{》) Nachteile des TAH sind die Implantatgröße und der deutlich erhöhte Geräuschpegel}

Nachteilig sind die Größe der Implantate, die im Querschnitt größeren Druckleitungen und der deutlich höhere Geräuschpegel der Antriebseinheit im Vergleich zu elektrischen Rotationspumpen [21].

Derzeitige Indikationen umfassen $\mathrm{Pa}$ tienten im Stadium IV nach NYHA oder im Stadium I, II oder III nach der Interagency Registry for Mechanically Assisted Circulatory Support (INTERMACS; [10]), die ein hohes Risiko haben, rasch an einem biventrikulären Herzversagen zu versterben. Weiterhin gehören alle Situationen dazu, in denen die Implantation eines univentrikulären - meist linksventrikulären - VAD kontraindiziert ist (-Infobox 1), zudem auch etliche komplexe kongenitale Vitien mit terminaler Kardiomyopathie [22].

Da bei der Implantation des TAH CardioWest ${ }^{\mathrm{tm}}$ beide nativen Ventrikel und alle 4 nativen Klappen entfernt werden, eliminiert man somit auch alle Probleme, die im Zusammenhang mit links- oder biventrikulären VAD beobachtet werden können: Rechtsherzversagen, Regurgitationen bei Klappeninsuffizienzen, maligne Arrhythmien, intraventrikuläre Kommunikationen und Low-flowSituationen [6].

\section{Implantation}

Die Implantation ist chirurgisch an sich einfach, für einen Herzchirurgen aber trotzdem herausfordernd, weil das native Herz größtenteils reseziert wird und der Eingriff damit irreversibel ist. Somit scheidet auch eine "bridge to recovery“ a priori aus. Unabdingbar ist bei der Verwendung der $70-\mathrm{cm}^{3}$-Ventrikel ein entsprechend großer Patient mit einer Körperoberfläche (KOF) $>1,7 \mathrm{~m}^{2}$ und einer a.p.-Distanz zwischen hinterem Sternum und Vorderkante des zehnten Brustwirbelkörpers $\geq 10 \mathrm{~cm}$, also mit einem großen Thorax [22]. Würde 
man das TAH CardioWest ${ }^{\mathrm{Tm}}$ in einen $\mathrm{zu}$ kleinen Patienten implantieren, könnte dies zu einer Kompression der V. cava inferior bzw. der linken Lungenvenen und somit nach dem Thoraxverschluss zu einer massiven Füllungsbehinderung der Pumpkammern mit deletären Konsequenzen führen.

Der operative Ablauf - auf wenige, essenzielle Punkte reduziert - beginnt nach medianer Sternotomie und Etablierung der extrakorporalen Zirkulation durch eine Herz-Lungen-Maschine (HLM) mit der Resektion des irreversibel erkrankten Herzens auf der Ventrikelseite oberhalb der AV-Grube, wobei ein schmaler muskulärer Ventrikelsaum erhalten bleibt. Mitral- und Trikuspidalklappe werden bis auf einen 2-mm-Saum reseziert, Aorta und Truncus pulmonalis oberhalb der Klappen am sinotubulären Übergang quer durchtrennt. Der Koronarsinus wird an seiner Mündung in den rechten Vorhof vernäht. Filzarmiert werden nun „inflow connectors" auf den muskulären Ventrikelsaum als atriale Cuffs aufgenäht. Mit diesen atrialen Manschetten müssen später die artifiziellen Pumpkammern konnektiert werden.

1) Bei allen Anastomosen muss auf eine absolute Hämostase geachtet werden

Die Ausflusstraktprothesen mit ihren Konnektoren werden in End-zu-EndTechnik mit der aszendierenden Aorta und dem Truncus pulmonalis anastomosiert. Auf eine absolute Hämostase muss bei allen Anastomosen geachtet werden, da sie nach Einbringen der Pumpkammern nicht mehr einsehbar sind und potenzielle Blutungen nachträglich nur mit extremem Aufwand saniert werden können (• Abb. 1).

Die mit physiologischer Kochsalzlösung gefüllten Ventrikel werden mit den atrialen Manschetten und den Ausflusstraktprothesen konnektiert (• Abb. 2), zuerst die linke, schließlich die rechte Pumpkammer. Die Druckleitungen werden durch die Bauchdecke in der Regio epigastrica ausgleitet und mit dem pneumatischen Antrieb verbunden. Nach sorgfältiger Entlüftung werden die
Wien klin Mag 2016 · 19:48-54 DOI 10.1007/s00740-016-0097-2

(c) The Author(s) 2016

\section{H. Antretter · J. Dumfarth · D. Höfer \\ Das komplett künstliche Herz}

\section{Zusammenfassung}

Hintergrund. Das komplett künstliche Herz [,"total artificial heart" (TAH)] CardioWest ${ }^{\mathrm{TM}}$ ist derzeit der einzige, klinisch verfügbare mechanische Herzersatz bei irreversiblem, biventrikulärem Herzversagen.

Fragestellung. Wie lauten die Indikationen und Kontraindikationen für das TAH? Wie gestalten sich die Implantation und postoperative Betreuung?

Material und Methoden. Neben einer Übersicht über die Anwendung des TAH beinhaltet der vorliegende Beitrag eine kurze Vorstellung der zwei Patienten mit TAHImplantation an unserer Klinik.
Ergebnisse. Der klinische Verlauf, die postoperative Rehabilitation, Komplikationen im System sowie Steuermöglichkeiten werden vorgestellt.

Schlussfolgerungen. Das TAH ist ein Implantat zur effektiven Behandlung von Patienten im irreversiblen kardiogenen Schock als Überbrückung zur Transplantation.

\section{Schlüsselwörter}

Terminale Herzinsuffizienz - Mechanische Kreislaufunterstützung · Biventrikuläre Dekompensation · Postoperative Versorgung . Herztransplantation

\section{Total artificial heart}

\section{Abstract}

Background. To date the CardioWest ${ }^{\mathrm{TM}}$ total artificial heart is the only clinically available implantable biventricular mechanical replacement for irreversible cardiac failure. Objectives. This article presents the indications, contraindications, implantation procedere and postoperative treatment. Material and methods. In addition to a overview of the applications of the total artificial heart this article gives a brief presentation of the two patients treated in our department with the CardioWest ${ }^{\mathrm{TM}}$.
Results. The clinical course, postoperative rehabilitation, device-related complications and control mechanisms are presented. Conclusion. The total artificial heart is a reliable implant for treating critically ill patients with irreversible cardiogenic shock. A bridge to transplantation is feasible with excellent results.

\section{Keywords}

Heart failure, terminal · Mechanical circulatory support - Biventricular heart failure, irreversible $\cdot$ Postoperative care $\cdot$ Heart transplantation
Pumpkammern niederfrequent angefahren, unter Überwachung mithilfe der transösophagealen Echokardiographie (TEE) wird über mehrere Minuten die Pumpfrequenz gesteigert, schließlich der Patient von der HLM entwöhnt. Volumen wird so verabreicht, dass ein zentralvenöser Druck von 12-15 mmHg erreicht wird und das TAH etwa 7 l/min pumpt. Die Füllung und Ejektion aus der Pumpe in den Kreislauf sind volumenund vorlastabhängig und nehmen mit ansteigenden atrialen Drücken zu, d. h. je höher die Vorlast, desto höher das Herzzeitvolumen - ähnlich der FrankStarling-Reaktion.

Nach Protaminisierung und Reetablierung einer normalen Gerinnung er- folgt der Thoraxverschluss in konventioneller Weise. Mittels TEE werden konzentriert die V. cava inferior und die Mündung der linken Lungenvenen beurteilt, um eine Kompression durch die Pumpkammern zu erkennen. Indem die Position der Pumpkammern korrigiert wird, kann dieses Problem vor der Cerclage des Sternums beseitigt werden.

\section{Postoperative Antikoagulation}

Als das TAH zu Beginn der 1990er-Jahre klinisch breiter implementiert wurde, war noch ein antikoagulatorischer Ansatz mit Multimedikation gefordert, den man individuell an den Patienten anpasste. Nach initialer postoperativer 


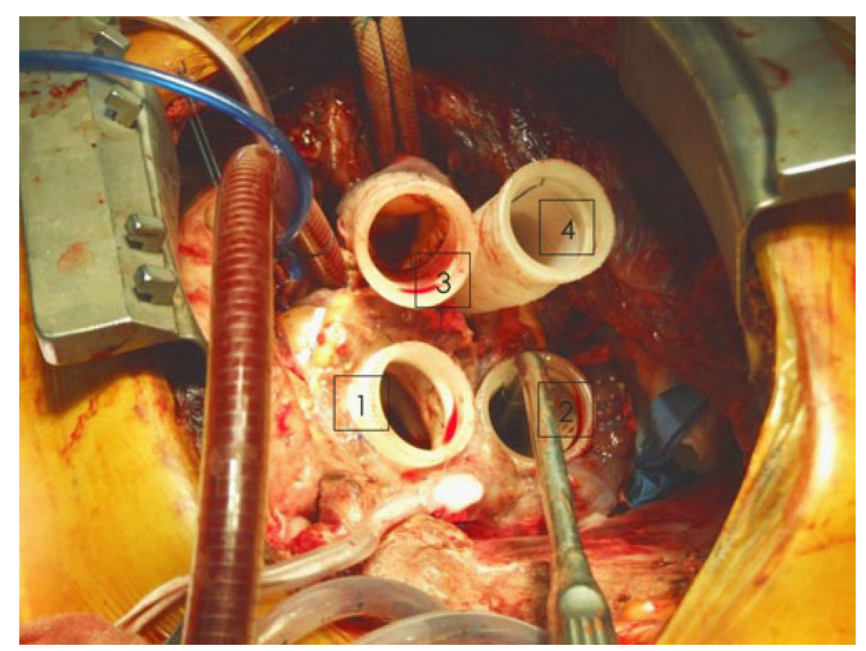

Abb. 1 A Intraoperativer Situs nach Resektion beider Ventrikel. Atriale Manschetten sind aufgenäht, ebenso Ausflusstraktprothesen an die Aorta ascendens bzw. den Truncus pulmonalis. Über diese Cuffs werden die künstlichen Ventrikel mit den Vorhöfen bzw. mit Aorta und Truncus pulmonalis konnektiert. 1 Rechte Vorhofmanschette; 2 linke Vorhofmanschette; 3 Prothese Aorta ascendens; 4 Prothese Truncus pulmonalis

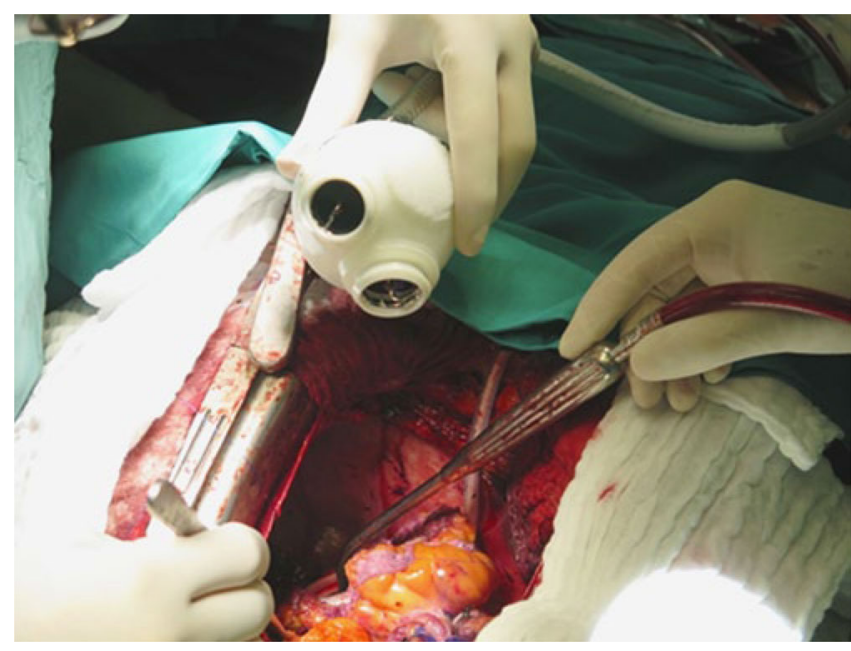

Abb. 2 \ Einbringen der linksventrikulären Pumpkammer in den Thorax. Gut ersichtlich sind die beiden Einscheibenprothesen in Mitral- und Aortenposition sowie die veloursbeschichtete Druckleitung, die später durch die Bauchdecke ausgeleitet wird
i.v.-Heparingabe erfolgte überlappend die Umstellung auf Vitamin-K-Antagonisten. Parallel wurde hoch dosiert Acetylsalicylsäure verabreicht, zudem als plättchenstabilisierende Substanz Dipyridamol und als antiinflammatorische Medikation Pentoxifyllin. Die Gerinnung wurde täglich mehrfach aufwendig kontrolliert, inklusive Thrombelastographie $[17,19]$.

\section{》) In den ersten $24 \mathrm{~h}$ nach der Operation erfolgt keine Gerinnungshemmung}

Heute ist die postoperative Antikoagulation unspektakulär: In den ersten $24 \mathrm{~h}$ nach der Operation werden keine gerinnungshemmenden Medikamente verabreicht! Danach wird unfraktioniertes Heparin kontinuierlich i.v. appliziert und eine aktivierte partielle Thromboplastinzeit (aPTT) von 55-65 s angestrebt. Acetylsalicylsäure wird ab dem zweiten bis vierten postoperativen Tag gegeben, die erwünschte Zieldosis liegt bei 1-bis 2-mal 100 mg/Tag. Nach Entfernung aller Thoraxdrainagen wird zu Vitamin-K-Antagonisten gewechselt; der Zielwert der International Normalized Ratio (INR) liegt bei 3-3,5. Die Heparingabe wird nach
Erreichen des therapeutischen INR-Bereichs beendet.

\section{Notfälle auf der Intensivstation}

Die typischen früh postoperativen Probleme auf der Intensivstation sind identisch zu denen aller artifiziellen Blutpumpen. So können Nachblutungen auftreten, die sich durch eine schlechter werdende Füllung der Ventrikel und damit durch eine Abnahme des Herzzeitvolumens bemerkbar machen abzulesen auf dem Display der Antriebskonsole (•Abb. 3). Eventuell kommt es zu einem Anstieg des zentralen Venendrucks aufgrund der Kompression intrathorakaler Strukturen. In der bildgebenden Diagnostik bei Verdacht auf eine Tamponade kommt die TEE oder eine Computertomographie (CT) des Thorax zum Einsatz (• Abb. 4).

Andererseits finden sich auch alle anderen Entitäten, die bei jedem Kollektiv schwerstkranker Patienten, oft mit entsprechenden Komorbiditäten, nach einer großen Herzoperation mit HLM beobachtet werden können, z. B. Sepsis, Multiorganversagen oder neurologische Komplikationen.

In der Frühphase nach Implantation auf der Intensivstation brauchen die Pumpkammern selbst in ihrer Ansteue- rung über das Display nicht verstellt werden, alle Parameter werden am Ende der Operation an der „stationären Konsole“ (Companion II) fix eingestellt (• Abb. 3.) und eigentlich nicht mehr verändert. Die Frequenz wird mit 130/min vorgewählt (Bereich: 120-140/min), die Systole mit $52 \%$ voreingestellt, ebenso werden der pneumatische Druck und das Vakuum für den linken und rechten Ventrikel vorjustiert (• Abb. 3.). Die Füllung der Ventrikel sollte bei $50-55 \mathrm{ml}$ liegen, etwa $75 \%$ des maximalen Ventrikelvolumens. Eine volumenbedingte Vorlasterhöhung führt automatisch zu einer Zunahme der Ventrikelfüllung bis zu einem Maximum von $70 \mathrm{ml}$, umgekehrt bedingt eine reduzierte Vorlast eine geringere Ventrikelfüllung unter $50 \mathrm{ml}$. Auf dem Display werden die Füllung und Druckentwicklung im Ventrikel auch graphisch in jeder Systole bzw. Diastole für beide Ventrikel angezeigt (•Abb. 3). Positive Effekte der hohen Pumpfrequenz sind u. a. die Vermeidung von Stasen im Ventrikel, deren optimale Auswaschung und die Vermeidung einer Thrombenbildung bzw. Embolie.

Die Mobilisation des Patienten erfolgt auf gleiche Weise wie die eines Patienten nach großer Herzoperation. Die Pflege der Durchtrittstellen der pneumatischen Druckleitungen durch die Bauchdecke 


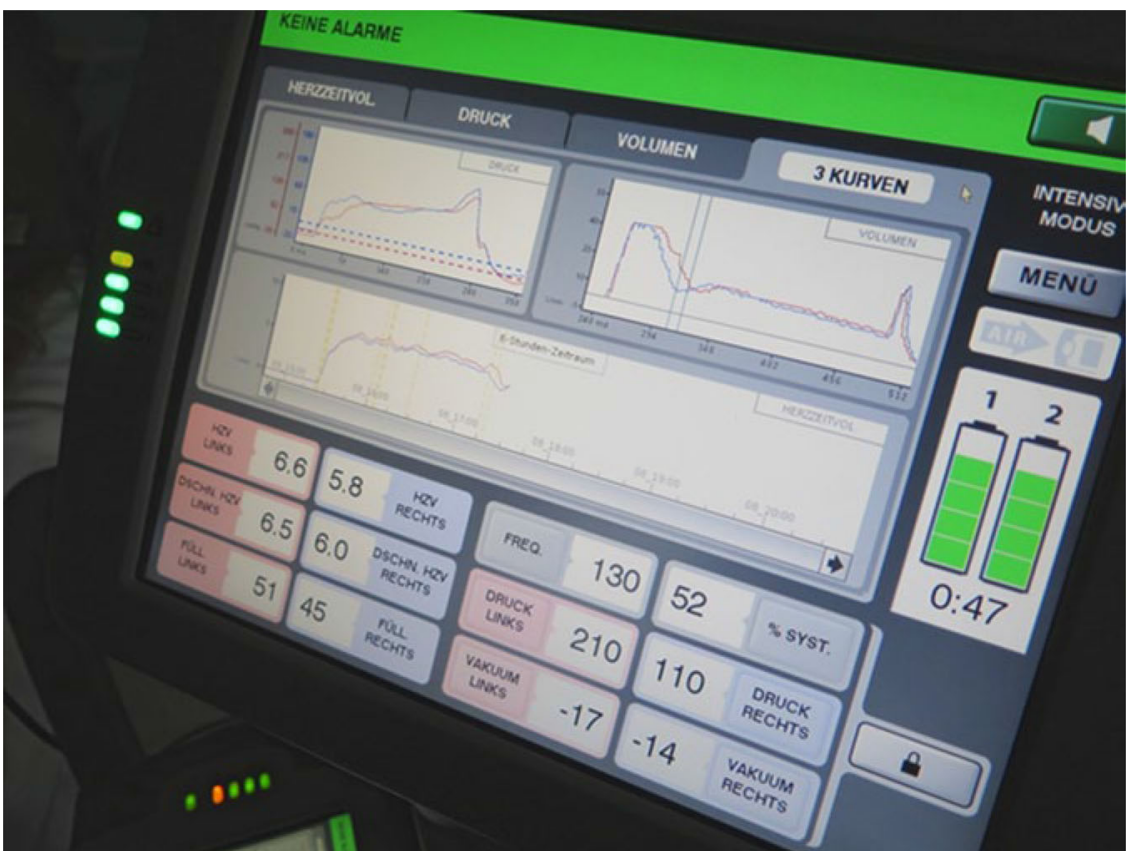

Abb. 3 A Display des Companion-II-Antriebs (innerklinische Verwendung). Rechts unten finden sich die Parameter, die intraoperativ eingestellt werden und praktisch nicht mehr verstellt werden (Frequenz, Systolendauer, Treibdruck und Vakuum für beide Ventrikel), links unten die variablen Parameter (Herzzeitvolumen und Füllung beider Ventrikel). Die drei Graphiken oben zeigen Druck und Volumen im rechten (blaue Kurve) und linken (rote Kurve) Ventrikel sowie das Herzzeitvolumen über die Zeit

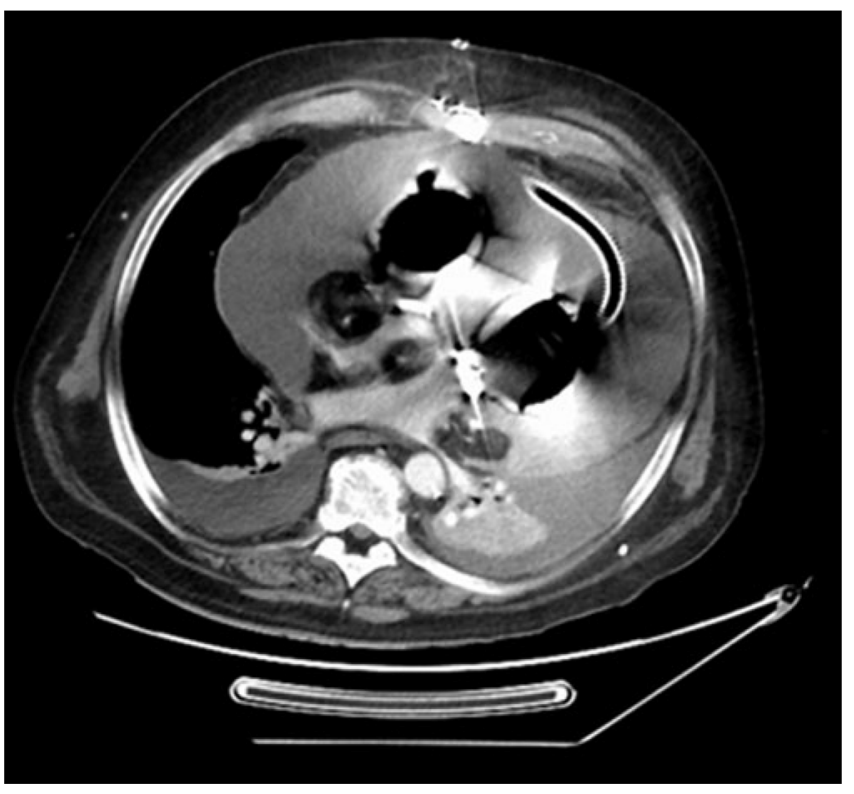

Abb. $4<$ Computertomographie des Thorax bei klinischen Zeichen einer beginnenden Perikardtamponade. Die artifiziellen Pumpkammern sind von einem breiten Hämatomsaum umgeben. Gut sichtbar ist auch der intrathorakale Verlauf der Druckleitung des linken Ventrikels. Nebenbefund: beidseits Pleuraergüsse

und die Prävention bzw. Therapie lokaler Infektionen werden in Analogie zu den „drivelines“ der VAD durchgeführt.

Besonders wichtig für die Intensivmedizin ist die Neuanlage von zentralvenösen Kathetern auf der Intensivstation, hier ist allergrößte Vorsicht geboten, da ein $\mathrm{zu}$ weit vorgeschobener SeldingerDraht oder zentralvenöser Katheter in der Inflow-Prothese des rechten Ventrikels stecken bleiben (Einklemmung) und zu einer Klappenblockade führen kann, was den Patienten in eine lebensbedrohliche Situation bringt; 3 Todesfälle sind in der Literatur beschrieben [7, 23]. Zentralvenöse Katheter dürfen bei CardioWest ${ }^{\text {TM }}$-Patienten nie den rechten Vorhof erreichen!
Notabene ist eine mechanische kardiopulmonale Reanimation bei einem Patienten mit implantiertem TAH CardioWest $^{\mathrm{Tm}}$ nicht zielführend, ebenso wenig das Schreiben eines Elektrokardiogramms (• Infobox 2)!

》) Die Patienten tolerieren eine niedrige Antikoagulation ohne zusätzliches thrombembolisches Risiko

Unter einer INR von 3-3,5 werden entsprechend mehr Blutungen auftreten. Lebensbedrohliche intrazerebrale Blutungen am TAH müssen im Sinne einer Nutzen-Risiko-Abwägung entsprechend rigoros therapiert werden, d. h., die Antikoagulation ist im Rahmen einer gravierenden Blutungssituation zu beenden. Die hohen Flüsse von $>61 / \mathrm{min}$ (auch im rechten Ventrikel) reduzieren offensichtlich das Risiko von Thromben, Patienten tolerieren Perioden einer niedrigen Antikoagulation ohne zusätzliches thrombembolisches Risiko.

Um eine INR $<2$ rasch $\mathrm{zu}$ erreichen, können Prothrombinkomplexgerinnungsfaktoren verabreicht werden (2000 IE initial, eventuell nachfolgend nochmals 1000 IE). Über $48 \mathrm{~h}$ kann dann jegliche orale Antikoagulation bzw. antiaggregatorische Therapie pausiert und eine aPTT von 40-55 s angestrebt werden; eine Beibehaltung über 4-5 Tage ist möglich. Nicht verabreicht werden sollte der rekombinante humane Blutgerinnungsfaktor VIIa (Novoseven ${ }^{\circledR}$ ).

Ist es in der ersten kranialen KontrollCT 48 h nach Auftreten der intrazerebralen Blutung zu keiner Befundverschlechterung gekommen, beginnt man mit einer niedrig dosierten i.v.-Gabe von Heparin (300 IE/h). Nach weiteren $48 \mathrm{~h}$ wird eine neuerliche kraniale CT durchgeführt. Ist wiederum keine Befundverschlechterung eingetreten, kann die aPTT auf 50-55 s, später dann auf 50-60 s gesteigert werden. In Analogie gilt dies auch für alle Formen gastrointestinaler Blutungen. Alle diesbezüglichen Angaben entstammen der persönlichen Kommunikation (Deutsches Herzzentrum Berlin; Herz- und Diabeteszentrum 


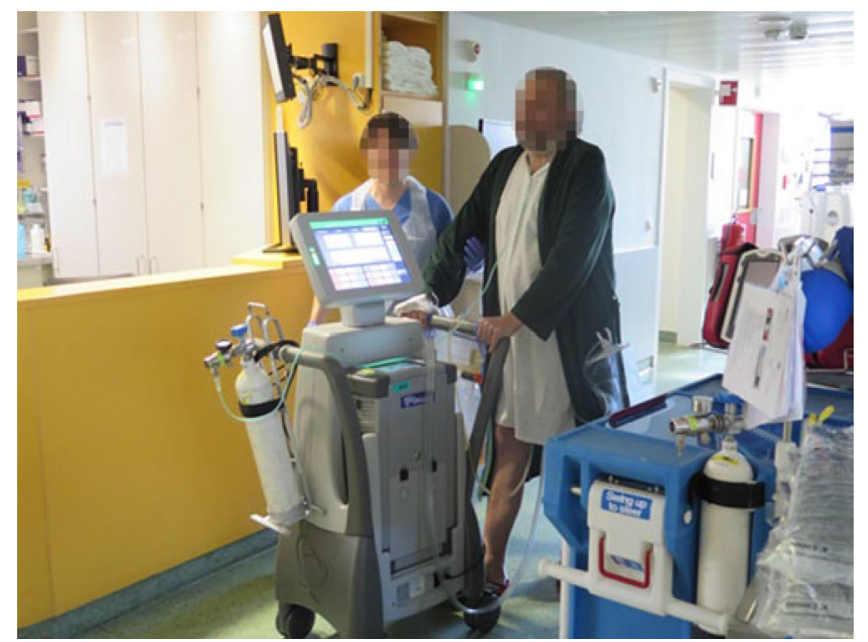

Abb. $5<$ Mobilisation des Patienten auf der Intensivstation am Companion-II-Driver (nicht am portablen "Freedom Driver", der nur $6 \mathrm{~kg}$ wiegt, außerklinisch zum Einsatz kommt und vom Patienten nicht in seinen Parametern verändert werden kann)

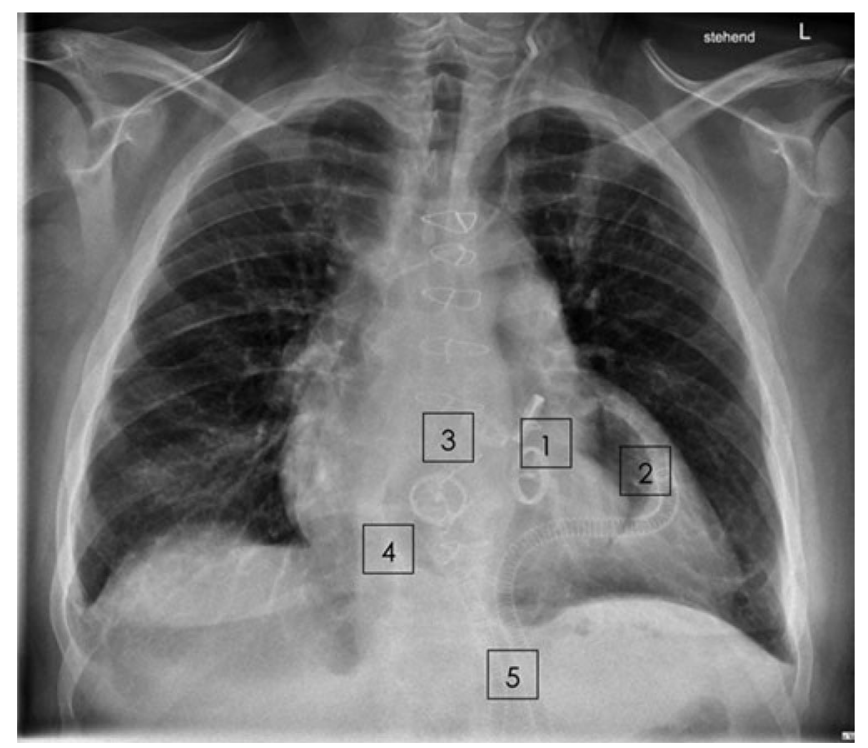

Abb. $6 \triangleleft$ Röntgenuntersuchung des Thorax am 78. postoperativen Tag vor Entlassung: 2 Kippscheibenprothesen (1) des linken Ventrikels (2); 2 Kippscheibenprothesen (3) der rechten Pumpkammer (4); Druckleitungen durch die Bauchdecke (5)

NRW, Bad Oeynhausen; Fa. SynCardia Systems, Inc., Tuscon, AZ, USA), zitierfähige Literatur gibt es diesbezüglich derzeit noch nicht.

\section{Eigene Erfahrungen}

Die Erfahrungen mit dem TAH CardioWest $^{\text {tix }}$ in Österreich sind sehr beschränkt. Wir haben an der Medizinischen Universität Innsbruck im Sommer 2008 den ersten Patienten in Österreich mit einem solchen System versorgt, im September 2014 schließlich den dritten Patienten Österreichs, den ersten, der die Klinik verlassen konnte und der nach Rehabilitation in der Sonderkrankenanstalt Rehabilitationszentrum Großgmain mobil zu Hause am System verweilt.
Die Indikation beim ersten Patienten war ein ausgedehnter transmuraler Vorderwandinfarkt mit malignen Arrhythmien und einem gescheiterten Weaning von der HLM nach 2-facher Bypassoperation. Sowohl die Implantation als auch der postoperative Verlauf gestaltete sich in Bezug auf das Kunstherz unproblematisch, am 14. postoperativen Tag verstarb der Patient jedoch bei schwerem Leberversagen.

Die Indikation bei unserem zweiten Patienten, der sehr groß und etwa $100 \mathrm{~kg}$ schwer ist, war ein schwerstes terminales biventrikuläres Versagen mit massiver Verkalkung des apikalen linken Ventrikels und großem intramuralem, apikalem Thrombus nach länger zurückliegendem Vorderwandinfarkt. Auch hier war die Implantation kompli- kationsfrei, der Patient rasch extubiert und der weitere postoperative Verlauf in Bezug auf das Kunstherz komplikationsfrei (• Abb. 5). Er befindet sich nun seit mehr als 290 Tagen ohne relevante Probleme am TAH (• Abb. 6) und ist seit Ende Januar 2015 zur kombinierten Herz-Nieren-Transplantation bei Eurotransplant gelistet.

Da die Parameter für die Steuerung weitgehend vorgegeben sind, sind Änderungen ähnlich den VAD kaum nötig. Die orale Antikoagulation ist einfach und standardisiert, unser Patient ist mit einem CoaguChek ${ }^{\circledR}$-Gerät (Fa. Roche) zu Hause versorgt und in der Selbstmessung der INR bzw. der Selbstmedikation seiner oralen Antikoagulation geschult. Störend bzw. teilweise sehr belastend wird die Geräuschentwicklung des portablen Antriebs vom Patienten bzw. seinen Angehörigen empfunden.

\section{Erfahrungen weltweit}

Bisher sind weltweit mehr als 1400 CardioWest ${ }^{\mathrm{T}} \mathrm{m}-\mathrm{TAH}$ implantiert worden. Die längste Implantationsdauer betrug 3,75 Jahre, ehe der Patient erfolgreich einer Transplantation unterzogen wurde. Insgesamt 47 Patienten befanden sich länger als ein Jahr am System, bei $72 \%$ war eine Transplantation möglich.

In der Bridge-to-transplant-Indikation überleben $70-80 \%$ der Patienten bis zur HTX, obwohl sie hauptsächlich zum Zeitpunkt der Implantation im Stadium INTERMACS I sind und damit zu den kränksten Patienten überhaupt zählen („crash and burn“, nur noch wenige Tage zu leben). Das 1-Jahres-Überleben nach erfolgter HTX liegt bei 80-86\% [4].

Schlaganfälle treten überwiegend in der Frühphase nach Implantation auf; die Häufigkeit liegt bei $4 \%$. Die Schlaganfallrate ab dem zweiten Tag nach der Implantation beträgt 8 Ereignisse auf 100 Patientenjahre bei Patienten, die keine DeviceInfektion aufweisen [3], und das sind immerhin $98 \%$. Fatale Device-Infektionen finden sich bei $2 \%$ der Patienten [4]. 
Infobox 2 Kontraindizierte bzw. nicht zielführende Verfahren bei komplett künstlichem Herz

- Zentralvenöse Katheter im rechten Vorhof

- Pulmonaliskatheter

- Mechanische kardiopulmonale Reanimation

- Externe elektrische Therapie beim Patienten (Defibrillation, Kardioversion, externer Pacer)

- Ableitung eines Elektrokardiogramms

\section{Neues System mit kleineren Ventrikeln}

Aufgrund der Tatsache, dass bisher nur Erwachsene mit einer KOF $>1,7 \mathrm{~m}^{2}$ mit einem CardioWest ${ }^{\mathrm{mt}}$-TAH versorgt werden konnten, hat die Firma SynCardia $50-\mathrm{cm}^{3}$-Ventrikel entwickelt und klinisch implementiert. Diese können nun in kleinere Patienten mit einer KOF von $1,2-1,7 \mathrm{~m}^{2}$ implantiert werden, seit Anfang 2015 sind sie CE-zertifiziert und klinisch verfügbar. Somit werden in Zukunft auch Kinder, Jugendliche und kleinere Erwachsene von dieser Form des mechanischen Herzersatzes bei irreversiblem, dekompensiertem biventrikulärem Herzversagen profitieren können.

Im Jahr 2013 wurden laut der INTERMACS-Registry in den USA $66 \mathrm{~Pa}-$ tienten mit einem TAH versorgt, was $2,7 \%$ der US-amerikanischen Population entspricht, die 2013 eine mechanische Herzunterstützung bzw. einen Herzersatz erhalten haben [14]. Durch die Implementierung einer zweiten Ventrikelgröße dürfte dieser Anteil in Zukunft deutlich zunehmen.

\section{Fazit für die Praxis}

- Das TAH CardioWest ${ }^{\mathrm{TM}}$ ist bei terminalem, therapierefraktärem biventrikulärem Herzversagen im NYHAStadium IV in der Indikation „bridge to transplantation" indiziert.

- Durch den radikalen chirurgischen Ansatz mit Resektion des erkrankten nativen Herzens werden viele Probleme derzeitiger biventrikulärer parakorporaler Systeme aufgrund ihrer Kanülierung vermieden.
- Herzminutenvolumina über 9 I sind möglich.

- Die postoperative orale Dauermedikation mit Acetylsalicylsäure und Cumarin ist unkompliziert, die ZielINR liegt bei 3-3,5.

- Zentralvenöse Katheter dürfen bei TAH-Patienten nie in den rechten Vorhof reichen, sonst besteht die Gefahr einer Blockade der InflowProthese des rechten Ventrikels mit deletären Folgen für den Patienten.

\section{Korrespondenzadresse}

Univ.-Prof. Dr. H. Antretter

Universitätsklinik für Herzchirurgie, Department Operative Medizin, Medizinische Universität Innsbruck

Anichstr. 35, 6020 Innsbruck, Österreich herwig.antretter@i-med.ac.at

\section{Einhaltung ethischer Richtlinien}

Interessenkonflikt. H. Antretter, J. Dumfarth und D. Höfer geben an, dass kein Interessenkonflikt besteht.

Alle Patienten, die über Bildmaterial oder anderweitige Angaben innerhalb des Manuskripts zu identifizieren sind, haben hierzu ihre schriftliche Einwilligung gegeben. Im Falle von nicht mündigen Patienten liegt die Einwilligung eines Erziehungsberechtigen oder des gesetzlich bestellten Betreuers vor.

Open Access. This article is distributed under the terms of the Creative Commons Attribution 4.0 International License (http://creativecommons.org/ licenses/by/4.0/), which permits unrestricted use, distribution, and reproduction in any medium, provided you give appropriate credit to the original author(s) and the source, provide a link to the Creative Commons license, and indicate if changes were made.

\section{Literatur}

1. Antretter H, Laufer G (2001) Surgical techniques for cardiac transplantation. Acta Chir Austriaca 33:17-24

2. Barnard J, Tsui SSL (2012) The total artificial heart in a cardiac replacement therapy programme. $\mathrm{Br} \mathrm{J}$ Hosp Med 73:634-639

3. Bonios MJ, Terrovitis JV, Drakos SG et al (2012) Comparison of three different regimens of intermittent inotrope infusions for end stage heart failure. Int J Cardiol 159:225-229

4. Copeland JG (2013) SynCardia total artificial heart. Update and future. Tex Heart Inst J 40:587-588

5. Copeland JG, Arabia FA, Tsau PH et al (2003) Total artificial hearts: bridge to transplantation. Cardiol Clin 21:105-117

6. Copeland JG, Smith RG, Arabia FA et al (2004) Cardiac replacement with a total artificial heart as a bridge to transplantation. N Eng J Med 351:859-867

7. El-Banayosy A, Arusoglu L, Morshuis Met al (2005) CardioWest total artificial heart: Bad Oeynhausen experience. Ann Thorac Surg 80:548-552

8. Fitzpatrick JR 3rd, Frederick JR, Hsu VM et al (2008) Risk score derived from pre-operative data analysis predicts the need for biventricular mechanical circulatory support. J Heart Lung Transplant 27:1286-1292

9. Hoefer D, Poelzl G, Kilo J et al (2005) Early detection and successful therapy of fulminant chlamydia pneumoniae myocarditis. ASAIO J 51:480-481

10. Holman WL (2012) Interagency Registry for Mechanically Assisted Circulatory Support (INTERMACS): what have we learned and what will we learn? Circulation 126:1401-1406

11. Jessup M, Brozena S (2003) Heart failure. N Eng J Med 348:2007-2018

12. Kirklin JK, Naftel DC, Pagani FD et al (2012) Long-term mechanical circulatory support (destination therapy): on track to compete with heart transplantation? J Thorac Cardiovasc Surg 144:584-603

13. Kirklin JK, Naftel DC, Kormos RL et al (2012) The fourth INTERMACS annual report: 4000 implants and counting. J Heart Lung Transplant 31:117-126

14. Kirklin JK, Naftel DC, Pagani FD et al (2014) Sixth INTERMACS annual report: a 10,000-patient database. J Heart Lung Transplant 33:555-564

15. Poelzl G, Frick M, Antretter H et al (2006) Interventional and medical treatment of acute heart failure due to inflammation. Herz 31:1761-1766

16. Puehler $T$, Ensminger $S$, Schoenbrodt $M$ et al (2014) Mechanical circulatory support devices as a destination therapy - current evidence. Ann Cardiothorac Surg 3:513-524

17. Roussel JC, Senage T, Baron O et al (2009) CardioWest (Jarvik) total artificial heart: a single center experience with 42 patients. Ann Thorac Surg 87:124-130

18. Stehlik J, Edwards LB, Kucheryavaya AY et al (2011) The registry of the International Society for Heart and Lung Transplantation: Twenty-eighth Adult Heart Transplant Report - 2011. J Heart Lung Transplant 30:1078-1094

19. Szefner J, Cabrol C (1993) Control and treatment of hemostasis in patients with a total artificial heart: the experience at La Pitie. In: Piffare R (Hrsg) Anticoagulation, hemostasis and blood preservation in cardiovascular surgery. Hanley and Belfus, Philadelphia, S237-264

20. Takeda K, Takayama H, Kalesan B et al (2015) Outcome of cardiac transplantation in patients requiring prolonged continuous-flow left ventricular assist device support. J Heart Lung Transplant 34:89-99

21. Tarzia V, Buratto E, Gallo M et al (2014) Surgical implantation of the CardioWest Total Artificial Heart. Ann Cardiothorac Surg 3:624-625

22. Torregrossa G, Anyanwu A, Zucchetta F, Gerosa G (2014) SynCardia: the total artificial heart. Ann Cardiothorac Surg 3:612-620

23. Zimmerman $\mathrm{H}$, Coehlo-Anderson R, Slepian Met al (2010) Device malfunction of the CardioWest total artificial heart secondary to catheter entrapment of the tricuspid valve. ASAIO J 56:481-482 